\title{
Teste de um equipamento para classificação de peças estruturais de madeira pela técnica da vibração transversal
}

\author{
An equipment test for grading lumber by \\ transverse vibration technique
}

Marcelo Rodrigo Carreira'; Marcela Candian ${ }^{2}$

\section{Resumo}

Devido à grande variabilidade de suas propriedades mecânicas, a racionalização no emprego da madeira para fins estruturais está diretamente condicionada a sua classificação. Existem diversas técnicas disponíveis para a classificação das peças estruturais de madeira, e dentre as atualmente empregadas, a de vibração transversal se salienta por obter resultados confiáveis na avaliação não destrutiva da madeira. Este trabalho tem por objetivo apresentar as bases para a classificação mecânica de peças de madeira e os resultados do teste de calibração do primeiro sistema de classificação por vibração transversal inteiramente fabricado no Brasil. A amostra utilizada nesta pesquisa era composta por 30 vigas de cupiúba (Goupia glabra) de dimensões nominais de $5 \mathrm{~cm}$ x $10 \mathrm{~cm}$ x $300 \mathrm{~cm}$. Os ensaios foram realizados no Laboratório de Madeiras e de Estruturas de Madeira (LaMEM) da Universidade de São Paulo (USP). Os resultados demonstram uma forte correlação entre o módulo de elasticidade medido pelo ensaio estático de flexão e o obtido com a avaliação pelo equipamento de vibração transversal, evidenciando a elevada confiabilidade do método para a classificação de peças estruturais de madeira. Obteve-se um coeficiente de determinação $\left(\mathrm{R}^{2}\right)$ de 0,896 com o equipamento produzido no Brasil. Este resultado demonstra que o aparelho pode ser utilizado na classificação de peças estruturais de madeira. Palavras-chave: Madeira. Estruturas de madeira. Classificação. Avaliação não destrutiva. Vibração transversal.

\begin{abstract}
Due to the great variability of its mechanical properties, the rational use of lumber for structural purposes is directly conditioned to its grading. There are several techniques available for grading structural lumber. The most relevant one is the transverse vibration technique which obtained reliable results in non-destructive evaluation of lumber. The purpose of this work is to present the bases for the mechanical grading of lumber and the results of the calibration test of the first transverse vibration equipment developed in Brazil. In this research 30 beams of cupiúba (Goupia glabra) with nominal dimensions of $5 \mathrm{~cm}$ X $10 \mathrm{~cm}$ X $300 \mathrm{~cm}$, were used. The tests were accomplished at the Wood and Timber Structures Laboratory (LaMEM) of the University of São Paulo (USP). The results showed a strong correlation between the elasticity modulus measured by the static bending test and the one obtained with the transverse vibration equipment, showing the high reliability of the vibration method for the grading of structural lumber. A determination coefficient $\left(\mathrm{R}^{2}\right)$ of 0.896 was obtained with the Brazilian equipment, showing that it can be used in the grading of lumber.
\end{abstract}

Key words: Lumber. Timber structures. Grading. Non-destructive evaluation. Transverse vibration.

\footnotetext{
1 Mestre em Engenharia de Estruturas, Universidade Tecnológica Federal do Paraná, Campus Campo Mourão. E-mail: carreira@ utfpr.edu.br.

2 Mestre em Engenharia, Universidade Federal de São Carlos. E-mail: marcela_candian@yahoo.com.br.
} 


\section{Introdução}

A madeira é um excelente material de construção, pois alia alta resistência mecânica, baixo peso, facilidade de ser trabalhada, beleza estética, menor emissão de poluentes para sua produção e, quando devidamente tratada, alcança grande durabilidade e pode ser utilizada nas mais diversas aplicações como revestimentos, esquadrias, e como material estrutural em fôrmas e cimbramentos para concreto armado, estruturas de pontes, coberturas. Entretanto, é um material encontrado na natureza e sua produção não se dá em uma indústria com rigorosos controles de qualidade, por isso a madeira caracteriza se pelo fato de ter grande variabilidade em suas propriedades mecânicas, e é, portanto, indispensável à realização da classificação estrutural desse material, de forma a garantir a sua utilização racional e tão segura quanto os demais materiais.

A classificação da madeira não tem por objetivo separar as peças de melhor qualidade e desprezar as de qualidade inferior, mas conhecer as propriedades mecânicas de cada peça, possibilitando o uso seguro de toda a capacidade resistente do material. Dessa forma, é possível projetar estruturas mais econômicas e com a mesma confiabilidade dos outros materiais estruturais.

A variabilidade observada nas propriedades mecânicas tem dois fatores de origem: o primeiro se deve à forte correlação entre a resistência mecânica e a densidade, havendo grande variação nessa característica de crescimento para as árvores de uma mesma espécie. O segundo corresponde ao efeito adverso que os defeitos, em particular os nós, provocam na resistência mecânica.

Atualmente existem diversas técnicas de avaliação não destrutiva aplicáveis à classificação estrutural da madeira. Entre elas, citam-se a: classificação visual, o ultra-som, o MSR (Machine Stress Rated), o raio $\mathrm{X}$, as ondas de tensão e a vibração transversal. Todas essas técnicas de avaliação não-destrutiva empregam um estimador para avaliar as propriedades mecânicas da madeira.
Na técnica de vibração transversal, a freqüência natural de vibração do material é correlacionada com sua rigidez na flexão.

De acordo com Ross e Pellerin (1994), a hipótese fundamental da avaliação não destrutiva da madeira foi fundamentada por Jayne (1959), segundo o qual a energia armazenada e as propriedades de sua dissipação na madeira podem ser medidas por meios não destrutivos, sendo tais mecanismos determinadores do comportamento estático do material.

Como conseqüência, podem ser obtidas convenientes relações matemáticas entre a resposta a fenômenos físicos e as propriedades estáticas de resistência e elasticidade da madeira, e isso permite a seleção das peças de melhor qualidade estrutural.

Recentemente, o engenheiro e professor Marcelo Rodrigo Carreira desenvolveu o primeiro equipamento produzido no Brasil para a classificação estrutural da madeira, utilizando a técnica da vibração transversal.

Este trabalho tem como objetivo investigar o embasamento teórico a respeito da classificação mecânica, bem como apresentar a calibração do equipamento de vibração transversal acima referido. A título de comparação, as peças também foram avaliadas utilizando o aparelho de vibração transversal E-computer modelo 340, do fabricante Metriguard.

\section{Revisão bibliográfica}

A classificação mecânica é o processo pelo qual a madeira é analisada por meio de um teste não destrutivo, seguido de uma inspeção visual para examinar certas características que a máquina não pode avaliar adequadamente. Ballarin, Targa e Palma (2002) explicam que a técnica de vibração transversal tem sido muito empregada especialmente pela forte aderência entre o modelo físico do fenômeno e o correspondente modelo teórico matemático. 
Para ilustrar o método, considere uma barra simplesmente apoiada como mostra a Figura 1.

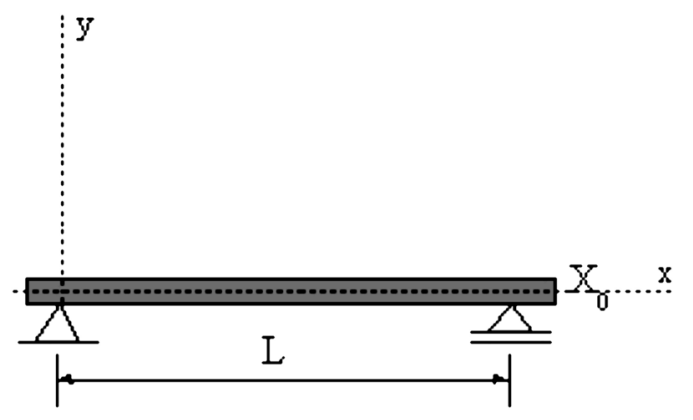

Figura 1. Barra simplesmente apoiada em repouso.

Quando a barra sofre uma perturbação mecânica causada por um impacto, o sistema passa a vibrar. Ao iniciar a vibração, uma força restauradora (Equação 1) atua no sistema, tendendo a levar a barra para a posição de equilíbrio (Figura 2)

$$
F=P_{0} \cdot \operatorname{sen}(\omega \cdot t)
$$

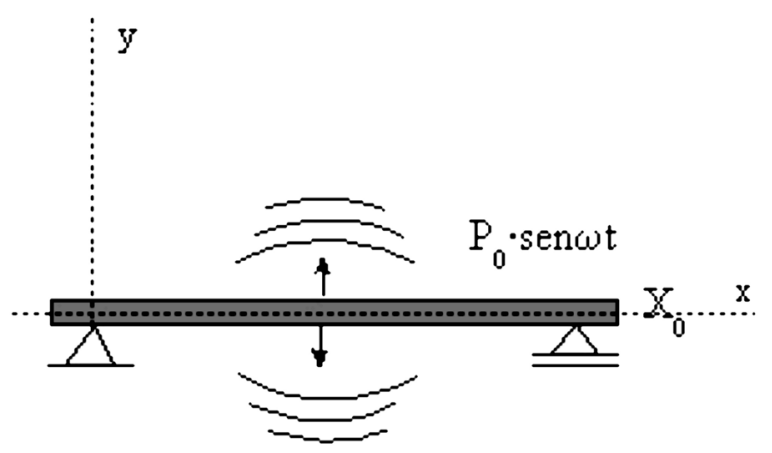

Figura 2. Ação da força restauradora na barra em vibração.

O modelo matemático que expressa esse comportamento é análogo ao do oscilador harmônico amortecido mostrado na Figura 3.

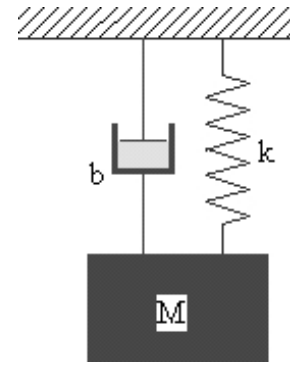

Figura 3. Oscilador harmônico amortecido.

O equacionamento teórico das vibrações transversais de uma viga é demonstrado por vários autores. Almeida (1999) e Seto (1964) apresentam a equação abaixo:

$$
\begin{aligned}
& E \cdot I\left(\frac{\partial^{4} y}{\partial x^{4}}\right)+\rho \cdot A \cdot \frac{\partial y^{2}}{\partial t^{2}}-\rho \cdot J \cdot\left(1+\frac{E}{\eta \cdot G}\right) \cdot \frac{\partial^{4} y}{\partial x^{2} \cdot \partial t^{2}}+ \\
& +\rho^{2} \cdot \frac{J}{\eta \cdot G} \cdot \frac{\partial^{4} y}{\partial t^{4}}=0
\end{aligned}
$$

Sendo:

E: módulo de elasticidade longitudinal do material; G: módulo de elasticidade transversal do material; y: deslocamento na direção y (perpendicular ao eixo da viga);

$\mathrm{x}$ : distância ao longo do comprimento da viga;

$\rho$ : densidade do material;

A: área da seção transversal;

t: tempo;

J: momento de inércia polar da seção;

I: momento de inércia da seção transversal.

Considerado as dimensões da seção transversal constantes e muito menores do que o comprimento da barra, e desprezado, ainda, a inércia à rotação e a influência do esforço cortante nas deformações, de modo que a Equação 2 se resume a:

$$
E \cdot I\left(\frac{\partial^{4} y}{\partial x^{4}}\right)+\rho \cdot A \cdot \frac{\partial y^{2}}{\partial t^{2}}=0
$$


Almeida (1999) apresenta a solução da equação diferencial acima para a primeira harmônica de uma viga simplesmente apoiada como:

$$
E=\frac{0,946 \cdot \rho \cdot A \cdot f^{2} \cdot L^{4}}{h^{2}}
$$

Sendo:

$\rho=$ densidade do material;

$\mathrm{fr}=$ freqüência de vibração;

$\mathrm{A}=$ área da seção transversal;

$\mathrm{L}=$ vão da peça;

$\mathrm{h}=$ altura da peça.

A relação entre o estimador e a propriedade mecânica de interesse é comumente mostrada por uma técnica estatística conhecida como regressão. A Figura 4 ilustra o uso de uma regressão linear para mostrar o efeito da variabilidade dos dados na precisão de uma avaliação.

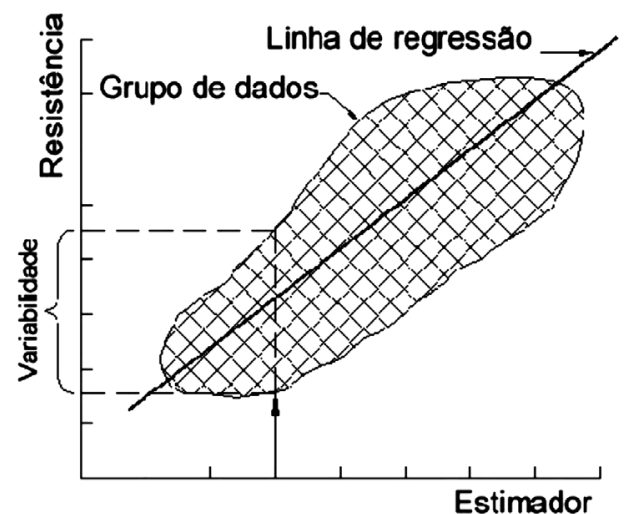

Figura 4. Estimativa da resistência por análise de regressão.

Se a linha de regressão for usada para estimar a resistência de uma peça de madeira, haverá uma probabilidade de $50 \%$ de a resistência da peça ser inferior ao valor estimado. Para que a regressão seja útil à inferência da resistência de novas amostras, é necessário determinar o intervalo de previsão (I.P.) da amostra.
Considerando-se, por exemplo, a linha inferior do I.P. de $90 \%$, garante-se com $95 \%$ de probabilidade que a resistência da peça classificada será maior do que a resistência estimada. De acordo com Galligan e Mcdonald (2000), os valores de resistência para o dimensionamento podem ser estimados dessa forma.

Como exemplo, a Figura 5 mostra o uso do módulo de elasticidade (MOE) como um estimador do módulo de ruptura à flexão (MOR). Nessa figura, ao invés da linha de regressão, a linha inferior do intervalo de previsão (I.P.) de $90 \%$ é usada para estimar o MOR. Esses dados se referem à madeira de pinus elliottii plantada na região de Lages, Estado de Santa Catarina.

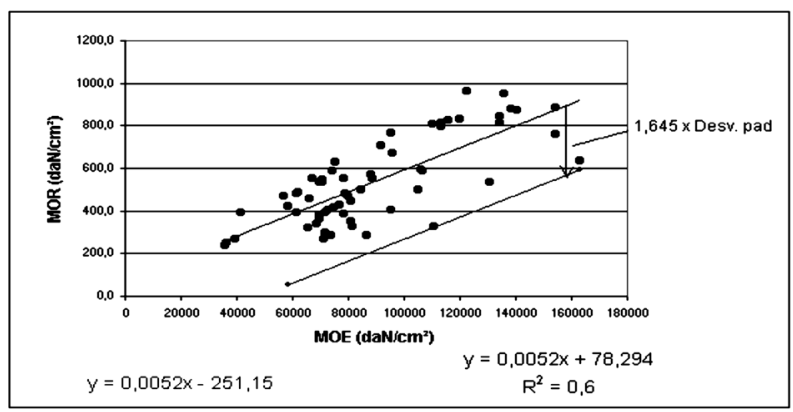

Figura 5. Relação típica entre o módulo de elasticidade e o módulo de ruptura.

Muitos trabalhos já foram desenvolvidos empregando a vibração transversal como técnica para a classificação da madeira, entre eles salientam-se:

a) Calil Júnior e Miná (2003) realizaram a classificação por vibração transversal em 326 peças estruturais de southern pine, cujas dimensões eram de $3,81 \mathrm{~cm} \times 13,97 \mathrm{~cm} \times 302,26 \mathrm{~cm}$. O teor de umidade das peças no momento do ensaio era de $12 \%$. Os autores utilizaram o aparelho E-computer 340 da Metriguard. Após o ensaio dinâmico, os autores realizaram o ensaio estático de flexão na amostra para a determinação do MOE estático. As peças foram simplesmente apoiadas e, no centro, aplicou-se uma força de 44,8N. Como resultado, obteve-se um coeficiente de correlação linear (r) 
de 0,98 entre o MOE estático e o MOE dinâmico e conclui-se que a vibração transversal é um método eficiente para a classificação da madeira.

b) Bartolomeu et al. (2004) desenvolveram um ensaio simplificado para a determinação do MOE pela técnica da vibração transversal. O sinal da vibração era captado por um microfone ligado à placa de som de um microcomputador e, para a determinação da freqüência de ressonância das peças, os autores empregaram um software para afinação de instrumentos musicais. A amostra era composta por 91 vigas de Angelim Araroba (Vataireopsis araroba) com dimensões de $6 \mathrm{~cm} \times 12$ $\mathrm{cm}$ x $250 \mathrm{~cm}$. Após o ensaio de vibração transversal, foi realizado o ensaio de flexão estática, para o qual foi utilizada a norma ASTM D 198-84 "Standard Methods of Static Test of Timber in Structural Sizes" (AMERICAN SOCIETY OF TESTING AND MATERIALS, 1997). Obteve-se o coeficiente de correlação linear (r) igual a 0,73 . Os autores concluem que o equipamento utilizado, apesar de simples, mostrou-se confiável.

c) Ballarin, Targa e Palma (2002) realizaram o teste de vibração transversal e também ensaios de flexão estática em 202 corpos-de-prova de dimensões $2 \mathrm{~cm} \times 2 \mathrm{~cm} \times 46 \mathrm{~cm}$, sendo 48 deles de Pinus taeda, 69 de Eucalipto citriodora, 57 de Eucalipto grandis e 28 de Eucalipto saligna. O equipamento utilizado para o ensaio de vibração transversal foi o BING (Beam Identification by Nondestructive Grading), produzido pelo CIRAD-Forest. Os coeficientes de correlação encontrados para a análise de regressão dos dados são 0,98 para o Pinus taeda, 0,87 para o Eucalipto citriodora, 0,87 para o Eucalipto grandis e 0,76 para o Eucalipto saligna. Os autores concluem que o método da vibração transversal revelou-se uma importante ferramenta para a classificação da madeira, e observam, que na média, o MOE dinâmico foi cerca de $12 \%$ superior ao MOE estático.

d) Green et al. (2004) realizaram a classificação de 120 toras, nas quais haviam misturadas as espécies subalpine fir (Albies lasiocarpa) e lodgepole pine
(Pinus contorta). O trabalho teve como objetivo o desenvolvimento de um embasamento técnico para a classificação mecânica de peças roliças usadas em log homes. Após a seleção da amostra, as toras foram torneadas para ficarem com a forma cilíndrica de aproximadamente $230 \mathrm{~mm}$ de diâmetro. $\mathrm{Na}$ seqüência, realizaram-se os testes de flexão estática e de vibração transversal. O coeficiente de determinação $\left(\mathrm{r}^{2}\right)$ encontrado foi de 0,95 .

e) Wang et al. (2001) realizaram os testes de flexão estática, vibração transversal e ondas de tensão em 159 toras de pequeno diâmetro, sendo 109 toras da espécie jack pine (Pinus banksiana Lamb.) e 50 da espécie red pine (Pinus resinosa Ait). O diâmetro da parte mais delgada das toras variou entre 127 a $310 \mathrm{~mm}$. O objetivo desse trabalho foi investigar o uso destas técnicas na avaliação do MOE de toras de pequeno diâmetro. Para a vibração transversal, os coeficientes de determinação entre o MOE estático e o MOE obtido com a técnica de vibração transversal foram 0,95 para o red pine e 0,85 para o jack pine. A conclusão dos autores é que o método de vibração transversal mostrou-se mais eficiente na avaliação do MOE do que o método das ondas de tensão, pois a técnica de vibração transversal apresentou-se menos sensível às imperfeições geométricas das toras.

\section{Materiais e métodos}

Neste trabalho, foram utilizadas 30 vigas de cupiúba com dimensões nominais de $5 \mathrm{~cm}$ x $10 \mathrm{~cm}$ x $300 \mathrm{~cm}$. As peças estavam armazenadas em local coberto.

\section{Determinação do teor de umidade}

O teor de umidade das peças foi determinado pelo ensaio de umidade, conforme anexo $\mathrm{B}$ da norma NBR 7190 "Projeto de Estruturas de madeira" (ASSOCIAÇÃO BRASILEIRA DE NORMAS TÉCNICAS, 1997). Para tanto, antes dos testes de flexão, retirou-se, aleatoriamente, uma amostra de 
seis corpos-de-prova para a determinação do teor de umidade. Os corpos-de-prova foram levados à estufa a uma temperatura de aproximadamente $100^{\circ} \mathrm{C}$ até atingirem a constância da massa.

Teste de vibração transversal com o equipamento nacional

O equipamento utilizado neste teste é produzido em Campo Mourão, Paraná, e é resultado de 5 anos de pesquisa do prof. Marcelo Rodrigo Carreira, com o método de avaliação não-destrutiva por vibração transversal. As pesquisas com a técnica de vibração transversal foram inteiramente financiadas com recursos próprios e desenvolvidas em seus horários de folga, nos finais de semana.

A célula de carga do equipamento foi calibrada utilizando-se para essa operação um peso de $50 \mathrm{~N}$. $\mathrm{O}$ equipamento emprega a Equação 4 para medir o módulo de elasticidade do material.

Antes de realizar o teste, foram feitas as medições das dimensões das peças utilizando-se uma trena metálica com resolução de $1 \mathrm{~mm}$.

As peças foram testadas com um vão livre de 2,90 m, segundo o eixo de menor inércia.

Para produzir a vibração nas peças, aplicou-se um leve golpe no centro do vão (Figura 6).

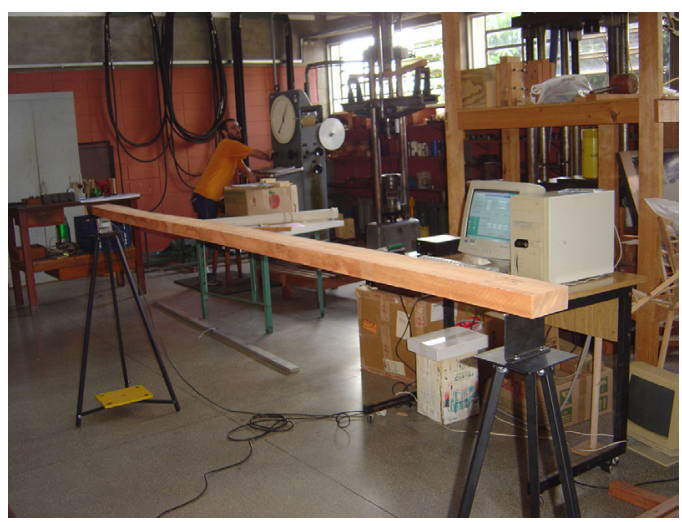

Figura 6. Equipamento de vibração transversal Carreira. desenvolvido pelo prof. Marcelo Rodrigo.
Teste de vibração transversal com o equipamento da metriguard

$\mathrm{O}$ teste realizado com o equipamento da Metriguard seguiu aos mesmos procedimentos do teste com o equipamento produzido por Carreira. A Figura 7 apresenta uma vista do ensaio com esse equipamento.

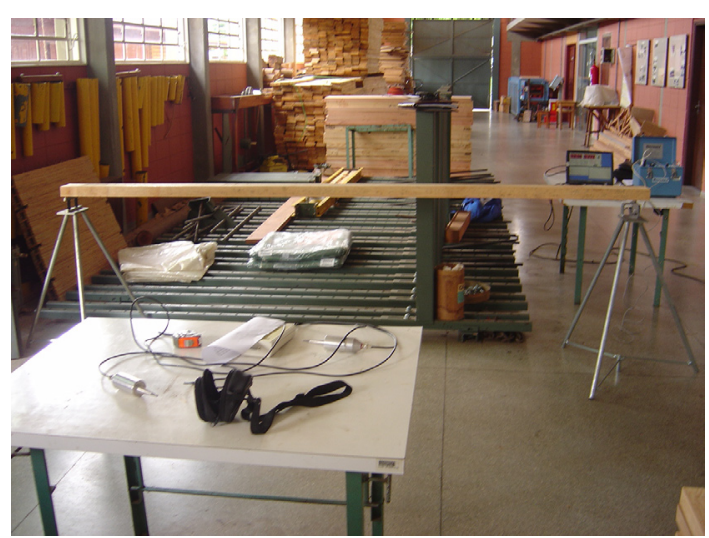

Figura 7. Equipamento E-computer modelo 340 da Metriguard.

Teste de flexão estática em relação ao eixo de menor inércia

O teste de flexão estática foi realizado aplicandose uma força concentrada no centro do vão das peças. Mediram-se os deslocamentos verticais em cinco níveis de carga, sendo: 70,6 N, 141,2 N, 211,8 $\mathrm{N}, 282,4 \mathrm{~N}$ e $353 \mathrm{~N}$. A força foi medida com um anel dinamométrico, com capacidade máxima de $4,70 \mathrm{kN}$. Utilizou-se um relógio comparador de resolução centesimal posicionado no centro do vão, para medir o deslocamento vertical provocado pela força (Figura 8). Para a aplicação da força, utilizouse um cilindro hidráulico com capacidade para $250 \mathrm{kN}$ e uma bomba manual da Yellow Power.

O módulo de elasticidade médio foi determinado pela média aritmética do módulo de elasticidade medido em cada nível de carga. 


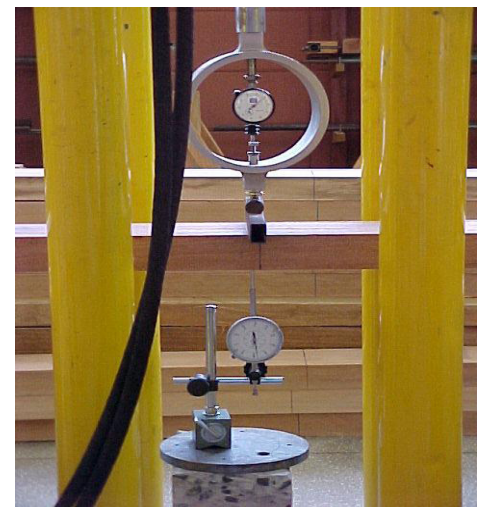

Figura 8. Teste de flexão estática em relação ao eixo de menor inércia das vigas.

\section{Resultados e discussões}

Observou-se que as vigas decupiúbaapresentaram um teor de umidade médio de $12,5 \%$.

Os resultados obtidos com o teste de vibração transversal e com o ensaio estático de flexão para a cupiúba são apresentados na Tabela 1 . Os valores do MOE foram corrigidos para o teor de umidade de $12 \%$.

Observa-se que o MOE médio obtido com a vibração transversal de Carreira é aproximadamente $10 \%$ maior do que o MOE medido com o equipamento da Metriguard.

Para verificar se há diferença entre os resultados dos dois equipamentos, realizou-se a análise de variância dos dados para testar a seguinte hipótese:

$$
\left\{\begin{array}{l}
H_{0}: M_{1}=M_{2}=M_{3}=0 \\
H_{1}: M_{1} \neq M_{2} \neq M_{3}
\end{array}\right.
$$

A análise estatística dos dados, para a média dos módulos de elasticidade para a espécie considerada, inicia-se com a verificação da adequabilidade do modelo, isto é, realizando a análise dos resíduos do modelo.

Portanto, para verificar a igualdade de variância, utilizou-se o gráfico dos resíduos contra os valores estimados (Figura 9). Analisando o gráfico, podese considerar a variância constante, pois os pontos estão distribuídos aproximadamente eqüidistantes em relação ao zero.

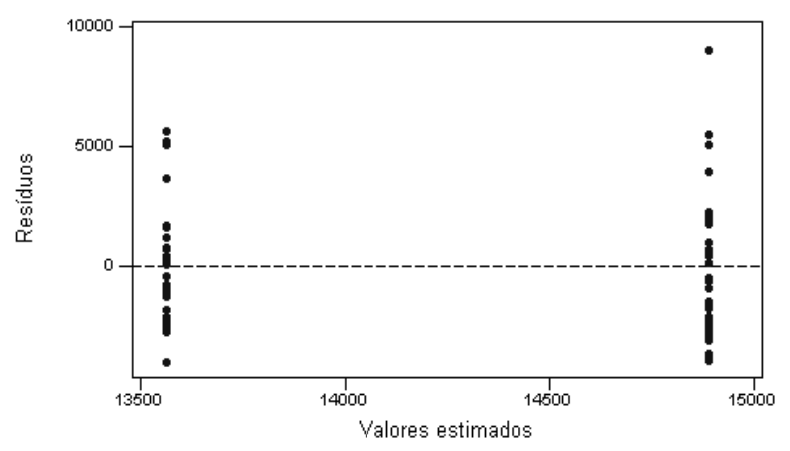

Figura 9. Gráfico dos resíduos contra valores estimados.

Tabela 1. Estatística descritiva dos dados da cupiúba.

\begin{tabular}{lrrr}
\hline & \multicolumn{3}{c}{ Tratamentos } \\
\cline { 2 - 4 } \multicolumn{1}{c}{ Estatística descritiva } & \multicolumn{1}{c}{$\mathrm{A}$} & $\mathrm{B}$ & $\mathrm{C}$ \\
& $M O E_{\text {estático }}$ & $M O E_{\text {Carreira }}$ & MOE \\
& $16.410,20$ & $14.890,29$ & $13.562,67$ \\
\hline Média (MPa) & $3.612,98$ & $3.039,55$ & $2.557,13$ \\
Desvio padrão (MPa) & $13.053 .631,19$ & $9.238 .844,18$ & $6.538 .895,19$ \\
Variância da amostra (MPa) & $22,0 \%$ & $20,4 \%$ & $18,9 \%$ \\
Coeficiente de variação (\%) & $11.322,56$ & $10.979,22$ & $9.563,23$ \\
Mínimo (MPa) & $24.160,73$ & $23.888,76$ & $19.226,93$ \\
Máximo (MPa) & 30 & 30 & 30 \\
Contagem & & & 30 \\
\hline
\end{tabular}


Para verificar se os resíduos apresentam distribuição normal, utilizou-se o teste de normalidade de Shapiro Wilk e concluiu-se que a suposição de normalidade não foi rejeitada pelos dados amostrais considerados, nos níveis usuais $(0,01<\alpha<0,05)$, conforme pode ser observado na Figura 10.

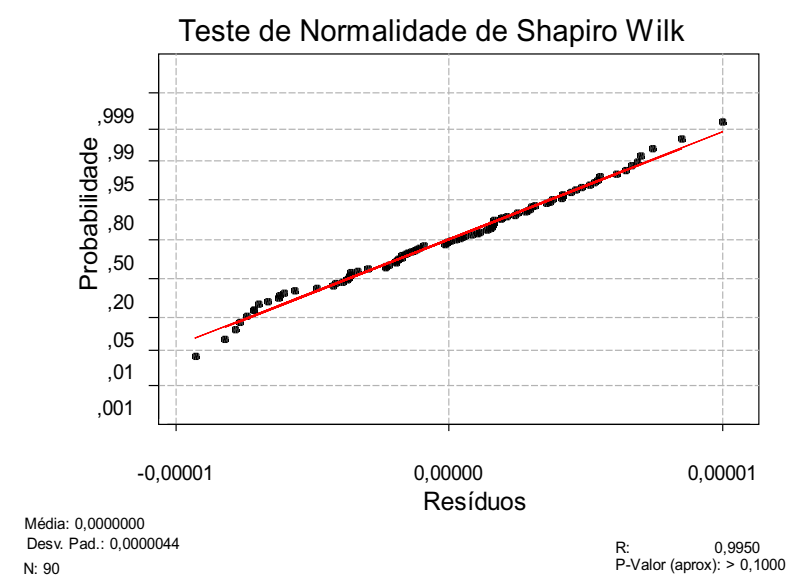

Portanto, da análise dos resíduos observa-se que o modelo da distribuição normal é adequado para análise dos dados, pois os pontos estão alinhados em relação à reta e além disso, o p-Valor é maior do que 0,05 . Dessa forma, a análise de variância pode ser utilizada para fazer inferências sobre as médias. A Tabela 2 apresenta o quadro de ANOVA (Análise de Variância).

Como $\mathrm{F}_{\text {cal }}=6,34>\mathrm{F}_{0,05 ; 2,87}=3,12$, rejeita-se $\mathrm{H}_{0}$ ao nível de significância $\alpha=0,05$. Ou seja, de acordo com os dados, a um nível de significância $\alpha$ $=0,05$, pode-se concluir que existe diferença entre as médias obtidas com os dois equipamentos.

Portanto, para verificar que as médias diferem entre si, foi utilizado o método de Dunnett. Este teste é empregado para averiguar se os resultados dos dois equipamentos apresentam diferença estatisticamente significativa em relação ao controle (módulo estático).

Figura 10. Teste de normalidade de Shapiro-Wilk.

Tabela 2. Análise de variância dos dados da cupiúba.

\begin{tabular}{lccccc}
\hline Fonte de variação & SQ & G.L. & QM & F cal & Valor-p \\
\hline Regressão & 121811267 & 2 & 60905634 & 6,34 & 0,003 \\
Erro & 836101766 & 87 & 9610365 & & \\
Total & 957913033 & 89 & & & \\
\hline
\end{tabular}

SQ: Soma dos Quadrados; G.L.: Graus de Liberdade; QM: Quadrado Médio; Fcal: F calculado; Valor-P: Valor de comparação com o nível de significância $\alpha$. 


\section{Teste de dunett}

Tomando $k=2, s^{2}=9610365 \Rightarrow s=3100,06 \mathrm{e}$ $v=8$, para $\alpha=0,05$, então, $t_{2,87,0,025}=2,25$ (critical value), a diferença mais significativa (d.m.s.) é:

$$
\begin{aligned}
& \text { d.m.s. }=t_{k, v, \alpha / 2} s \sqrt{\frac{1}{n_{C}}+\frac{1}{n_{i}}}= \\
& =2,25 \sqrt{\left(\frac{1}{30}+\frac{1}{30}\right) 9.610 .365}=1.800,97
\end{aligned}
$$

Os resultados das comparações múltiplas pareadas são apresentados na Tabela 3.

As diferenças estatisticamente significantes estão salientadas na Tabela 3 com dois asteriscos (**). Isto é, só o equipamento da Metriguard apresentou diferença estatisticamente significativa no valor do módulo de elasticidade médio em relação ao módulo obtido pelo ensaio estático de flexão. Esse resultado surpreendeu as expectativas em relação ao equipamento produzido, pois acreditava-se que ele apresentaria maior diferença estatística em relação ao módulo de elasticidade estático.

\section{Calibração do equipamento nacional}

A Figura 11 mostra o gráfico de dispersão e a reta de regressão entre os dados do MOE medido nos testes de flexão estática e pelo equipamento de vibração transversal com a cupiúba.

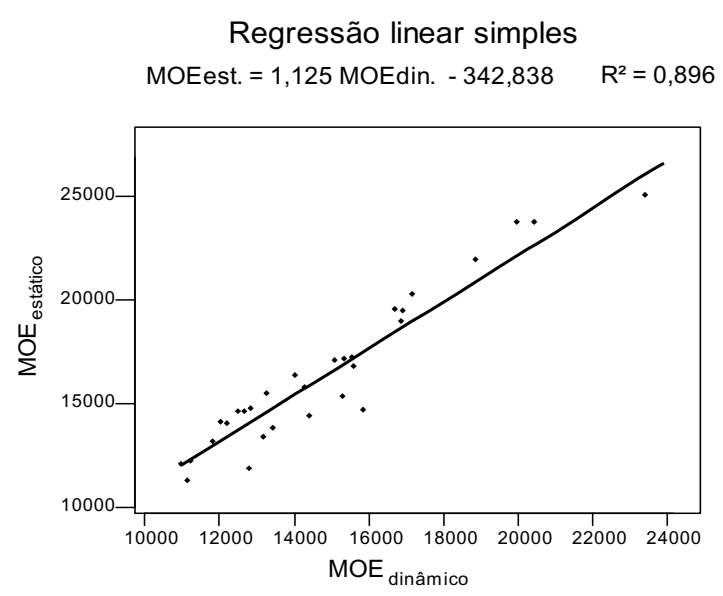

Figura 11. Diagrama de dispersão e reta de regressão entre MOE estático e MOE obtido com o equipamento vibração transversal desenvolvido por Carreira.

A Tabela 4 apresenta o quadro de ANOVAutilizado para verificar a validade do modelo ajustado.

Tabela 3. Comparações múltiplas pareadas para as médias.

\begin{tabular}{cccc}
\hline Tratamento & A (estático) & B (Carreira) & C (Metriguard) \\
\hline Média $\left(\bar{y}_{t}\right)$ & 16410 & 14890 & 13563 \\
Diferença $\left(\bar{y}_{i}-\bar{y}_{j}\right)$ & - & 1520 & $2847 * *$ \\
\hline
\end{tabular}

Tabela 4. Quadro de ANOVA para o MOE estático e o MOE obtido com a vibração transversal da cupiúba.

\begin{tabular}{lccccc}
\hline Fonte de variação & SQ & G.L. & QM & $F_{\text {cal }}$ & Valor-p \\
\hline Regressão & 339148483 & 1 & 339148483 & 241,03 & 0,000 \\
Erro & 39398277 & 28 & 1407081 & & \\
Total & 378546760 & 29 & & & \\
\hline
\end{tabular}

SQ: Soma dos Quadrados; G.L.: Graus de Liberdade; QM: Quadrado Médio; Fcal: F calculado; Valor-P: Valor de comparação com o nível de significância $\alpha$. 
Como $\mathrm{F}_{\text {cal }}=241,0300>\mathrm{F}_{0,05 ; 1,28}=4,20$, rejeitase $\mathrm{H}_{0}$ ao nível de significância $\mathrm{a}=0,05$. Ou seja, de acordo com os dados, a um nível de significância $\alpha=0,05$, pode-se concluir que existe evidência estatística de que a proporção da variância total explicada pela equação de regressão é altamente significativa. Portanto, o modelo de regressão linear dado pela Equação 7 é adequado para representar a relação entre o MOE estático e o MOE obtido com o equipamentodevibraçãotransversaldesenvolvidopor Carreira, para a espécie e dimensões consideradas.

$$
\mathrm{MOE}_{\text {estático }}=1,125 \mathrm{MOE}_{\mathrm{v}}-\mathbf{3} 42,838
$$

Observa-se que o coeficiente angular da reta de regressão é muito próximo de 1,0, indicando a elevada exatidão do equipamento de vibração transversal para a avaliação não destrutiva de peças estruturais de madeira.

O coeficiente de determinação $\left(\mathrm{R}^{2}\right)$ obtido foi de 0,896 . Comparando o $\mathrm{R}^{2}$ obtido com os valores apresentados na revisão bibliográfica, observa-se que a qualidade do ajuste foi boa.

\section{Conclusões}

A técnica de vibração transversal mostrou-se de rápida e de boa exatidão na avaliação da madeira.

Comparando o coeficiente de determinação encontrado $\left(R^{2}=0,896\right)$ com as pesquisas anteriores, pode-se afirmar que o equipamento produzido por Carreira apresentou bons resultados, pois o coeficiente de determinação encontrado foi maior do que os encontrados em alguns trabalhos.

A equação de calibração do equipamento desenvolvido por Carreira tem coeficiente angular de 1,12 , ou seja, muito próximo de 1,00, indicando que os valores do módulo de elasticidade obtidos com o equipamento desenvolvido são próximos do módulo estático.
Os resultados encontrados pelo equipamento desenvolvido por Carreira não demonstraram diferença estatisticamente significativa em relação do módulo de elasticidade estático. Portanto, concluise que o equipamento brasileiro tem boa exatidão e pode ser utilizado na determinação do módulo de elasticidade de peças estruturais de madeira.

O equipamento permitiu ainda a classificação rápida e das peças analisadas, cerca de 10 segundos por peça, mostrando-se adequado para a aplicação na indústria madeireira.

\section{Agradecimentos}

Os autores agradecem ao Laboratório de Madeiras e de Estruturas de Madeira (LaMEM) da Universidade de São Paulo (USP) por ceder os equipamentos utilizados nos ensaios de flexão estática e o equipamento E-Computer 340.

\section{Referências}

ALMEIDA, M. T. Análise de vibrações pelo método das matrizes de transferência. In: _. Vibrações mecânicas para engenheiros. 2. ed. São Paulo: Edgard Blücher, 1999.

ASSOCIAÇÃO BRASILEIRA DE NORMAS TÉCNICAS. NBR 7190 - Projeto de estruturas de madeira. Rio de Janeiro: ABNT, 1997.

AMERICAN SOCIETY OF TESTING AND MATERIALS. ASTM D198-84 - Standard Methods of Static Test of Timber in Structural Sizes. West Conshohoken, PA: ASTM, 1997.

BALLARIN, A. W.; TARGA, L. A.; PALMA, H. A. L. Ensaios não-destrutivos de vibração transversal na avaliação do módulo de elasticidade de madeiras de reflorestamento. In: ENCONTRO BRASILEIRO EM MADEIRAS E EM ESTRUTURAS DE MADEIRA, 8., 2002, Uberlândia, MG. Anais... Uberlândia: Universidade Federal de Uberlândia, 2002.

Ensaios não-destrutivos de vibração transversal na avaliação do módulo de elasticidade de madeiras de reflorestamento. In: VIII EBRAMEM - ENCONTRO BRASILEIRO EM MADEIRAS E ESTRUTURAS DE MADEIRA, 2002, Uberlândia - MG. Anais do VIII EBRAMEM, 2002. 
BARTOLOMEU, A. et al. Metodologia simplificada para o ensaio de vibração transversal em vigas estruturais de Angelim Araroba. ENCONTRO BRASILEIRO EM MADEIRAS E EM ESTRUTURAS DE MADEIRAS, 10., 2004, Cuiabá, MT. Anais... Cuiabá: UFMT, 2004. p. 29-29.

CALIL JÚNIOR, C; MINÁ, A. J. S. Vibração transversal: Um método eficiente para classificação de peças estruturais de madeira. Revista Brasileira de Engenharia Agrícola e Ambiental, Campina Grande, v.7, n.2: p.335338, 2003.

JAYNE, B. A. Vibrational properties of wood as indices of quality. Forest Products Journal, Madison, v. 9, n. 11, p. 413-416, 1959.

GALLIGAN, L. W.; MCDONALD, K. A. Machine grading of lumbe - Practical concerns for lumber producers. Madison: U.S.D.A Department of Agriculture, Forest Service, 2000. (General Technical Report FPLGTR-7).
GREEN, D. W; GORRNAN, T. M.; EVANS, J. W; MURPHY, J. F. Improved grading system for structural $\operatorname{logs}$ for log homes. Forest Products Journal, Madison. v. 54, n. 9, p. 59-62, 2004.

ROSS, R. J.; PELLERIN, R. F. Nondestructive testing for assessing wood members in structures. Madison: U.S.D.A Department of Agriculture, Forest Service, 1994. (General Technical Report FPL-GTR-70).

SETO, W. W. Vibrações transversais de vigas. In: $\overline{\text { HILL, } 1964 .}$ Vibrações mecânicas. São Paulo: McGRAW

WANG, X.; ROSS, R. J.; MATTSON, J. A.; ERICKSON, J. R.; FORSMAN, J. W.; GESKE, E. A.; WEHR, M. A. Several nondestructive evaluation techniques for assessing stiffnes and MOE of small-diameter logs. Madison: U.S.D.A Department of Agriculture, Forest Products Laboratory, 2001. (General Technical Report FPL-RP-600). 
